\title{
Bilayer Graphene Conductance Analysis based on FET Channel
}

\author{
Vahdat Nazerian ${ }^{1}$, Mohammad Javad Kiani ${ }^{2}$ \\ ${ }^{1}$ Department of Electrical Engineering, University of Mazandaran, Babolsar, Iran \\ ${ }^{2}$ Department of Electrical Engineering, Islamic Azad University, Yasooj Branch, Yasooj, Iran
}

\begin{tabular}{l}
\hline \hline Article Info \\
\hline Article history: \\
Received Jul 4, 2018 \\
Revised Jul 29, 2018 \\
Accepted Sep 6, 2018 \\
\hline
\end{tabular}

\section{Keyword:}

Band gap energy

Bilayer grapheme

Conductivity

FET channel

Resistance

\begin{abstract}
Graphene is considered as a famous nanomaterial because of some parameters such as its large surface-to-volume ratio, high conductivity, high mobility, and strong mechanical and elasticity properties. Therefore, in this work the conductance of two dimensional bilayer graphene (BG) is developed using the Fermi Dirac distribution function. For bilayer graphene two, various stacking structures (AA and $A B$ ) have been reported, which have armchair edge. Quantum gradient emerged between the channel and the gate and carrier movement of bilayer graphene is considered as FET channel, which is an important property of FET. Besides, band gap energy and resistance of BG have been modelled in this study. The impact of temperature on the resistance is extensively studied. It is demonstrated that the resistance of BG is the function of temperature and the conductance is increased at higher values of temperature.
\end{abstract}

Copyright $\left(C_{0} 2018\right.$ Institute of Advanced Engineering and Science. All rights reserved.

\section{Corresponding Author:}

Vahdat Nazerian,

Department of Electrical Engineering,

University of Mazandaran,

Babolsar, Iran.

Email: v.nazerian@umz.ac.ir

\section{INTRODUCTION}

The growing interest in two dimensional graphene has attracted much attention attributed because of its unique properties such as high mobility, its large surface-to-volume ratio, high conductivity and strong mechanical and elasticity properties [1]-[3]. Carbon base material with low dimensional behavior has been explored by many researchers for it has been widely used to accommodate nowadays technology. Recent research on the stability of few-layer, multilayer and even bilayer graphene [2]-[4] has been a brilliant founding. Also, for bilayer graphene nanoribbons (BGNs) two various stacking structures (AA and AB) have been reported, which have armchair edge [5], [6]. This study has focused on the AB-stacked configuration whose band gap is $0.02 \mathrm{eV}$ (which by applying vertical potential can make it to semiconductor) and is considered as semiconductor material, while the AA-stacked configuration is metallic [7], [8]. The atoms $\mathrm{A}_{2}$ and $\mathrm{B}_{2}$ existing in the $\mathrm{AB}$ structure of bilayer graphene were on the upmost layer of BGNs having hezgonal carbon lattice as shown in Figure 1, but atoms A1 and B1 exist on the lowest layer of them [9]-[11].

If the precise electric field is utilized on BGNs, a gap will be created on its band energy. By using voltage, it is possible to manage the value of band gap energy [12]. On the other hand, applied electric field effect can be observed in the form of carrier velocity in the channel region of a FET [13]. However, theoretical studies on the carrier velocity of BGNs needs to be done, and velocity characteristic based on the BGN band structure is needed to more explored.

Many researches and examinations have been lately conducted on GNR based FET. Fabrication too narrow nanowire, which has an appropriate energy band in such temperature is the most important challenge related to this issue [14]. These theoretical researches revealed that there is a negative relationship between width and the energy band gap of graphene nanoribbon. By decreasing the width of graphene, it make to graphene nanoribbon with only a couple of atoms of carbons, which it has a small bandgap. In the other 
words graphene with metallic properties make to graphene nanoribbon with semiconductor properties [15]. They also proved that for operating the transistor at room temperature while creating the smallest band gap, it is crucial to have width down to sub-10nm. However, in defining the function of the materials, the significant influence of edge effect and width must not be ignored [16].

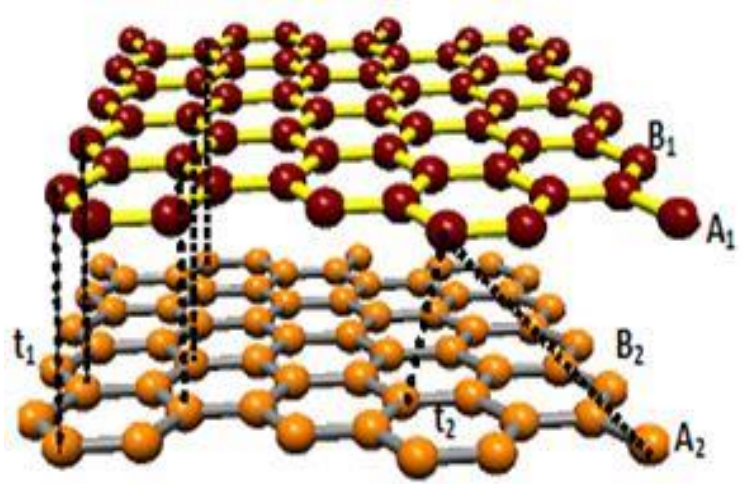

Figure 1. Schematic of AB bilayer graphene lattice configuration

This type of graphene has a limited width, so it is proposed as a one-dimensional material in our model [17]. Thus, some energy gap will be created. Therefore, the carriers in GNRs will be limited into a system with a single dimension, which this type of graphene can be applied to set structure devices [18]. In such conditions, there is an influential energy gap in one dimensional graphene nanoribbon structure. Where, $\mathrm{m}=3 \mathrm{p}$ and $\mathrm{m}=3 \mathrm{p}+1$, Armchair Graphene nanoribbons are semiconductors, and where $\mathrm{m}=3 \mathrm{p}+2$, they are metallic while $\mathrm{m}$ signifies a number of dimmer lines and $\mathrm{p}$ signifies an integer as depicted in Figure 2 . In addition, according to the available literature, there is a negative relationship between the widths of GNR and its energy gap mentioned before [19].

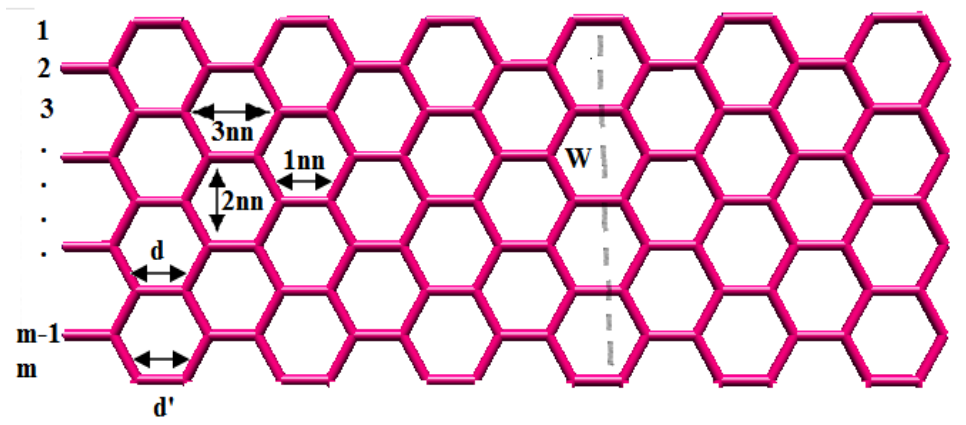

Figure 2. The schematic sketch of an AGNR

GNRs can be more considered as materials for integrated circuit interconnect than copper due to their low noise effect, thermal conductivity and the great electrical properties. Many experts and technologists are interested in GNRs because of their high quality [20]. So large numbers of studies have been conducted in this area and the relevant areas to gain more knowledge about them and their usage in these areas. A very related area is the construction of graphene based nanosensor [21].

\section{PROPOSED MODEL}

Figure 3 depicts a device sketch that includes a ballistic device attached to two contacts. It shows that in the two contacts there is a strong scattering according to which the thermal equilibrium is preserved, while no scattering is observed in the ballistic device [22], which we assumed to $A B$ stacking in our model as shown in Figure 3. 


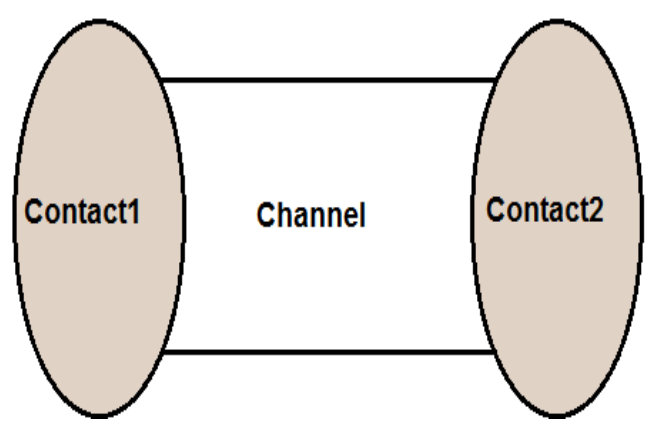

Figure 3. Sketch of a ballistic device with two contacts that function as reservoirs of thermal equilibrium carriers

A thermal equilibrium flux is injected into the device by contact 1 (the source). A number of the injected flux reflects from the possible barriers existing inside the device, the remaining flux passes through it and reach into contact 2 (the drain). Each electron incident on the contact is observed and thermalized making the contacts to be considered as perfect absorbers. Likewise, a thermal equilibrium flux is injected into the device by the drain. A number of injected flux is reflected through the possible barriers inside the device and the remaining of them pass through the source. Tunnelling and quantum reflections have been ignored in this section and electrons have been considered as semi-classical particles. It was intended to calculate the net current inside this device and the total density of electrons.

So, a semi-classical description has been employed according to which the local density-of-states inside the device equals only the local density-if-sates of bulk semiconductor, while shifted by the local electrostatic potential. If the changes in the electrostatic potential were not extremely fast, the function of the approximation would be considered good that would make it possible to neglect the quantum effects.

The studies illustrate that different layers of the graphene as a unique material system have an exceptional potential for FETs device applications [23]. In this regard, recent studies have shown that BLG with a gate-tunable band gap is the well-known material system for semiconductor application [24], [25]. Theoretically, band gap can be induced in grapheme with two methods. The first method is narrowing the graphene to nano-ribbon in which the band gap has a reverse relation with the width of nano-ribbon [26]. The second one is applying a perpendicular electric field on BLG that provides a potential difference between layers which opens the band gap in BLG [27]-[29]. This property makes it possible for BLG to be used in the future generation nano-electronic devices such as FETs. Figure 4 depicts BLG with a potential difference between the layers.

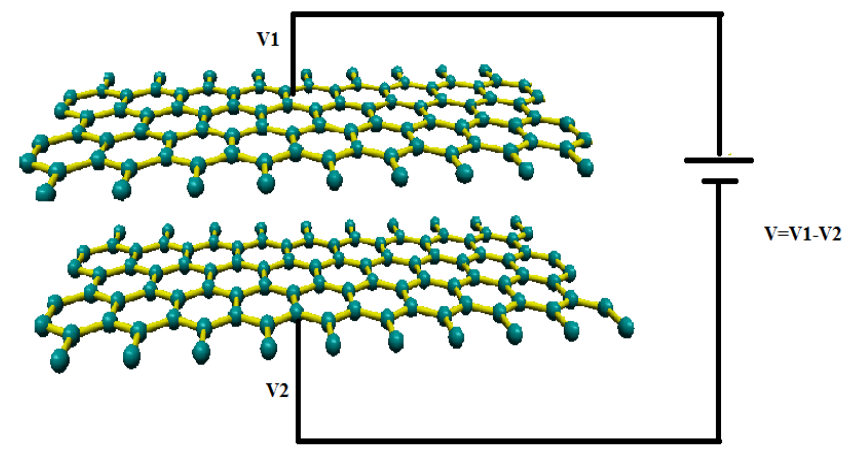

Figure 4. Perpendicularly applied voltage between top and bottom layers of BLG (biased BLG)

The unique electronic properties of BLG pave the way for its application [6]. As shown in Figure 4, in order to deform the BLG from a gapless system to a semiconductor material, an external voltage can be applied between the first and second layer to control the energy gap [5].

The unbiased configuration of BLG with AB stacking has a metallic performance, whereas its perpendicularly applied voltage configuration has a semiconductor property with a band gap shown in Figure 
5 [30]. Energy-wave vector characteristic of biased BLG can be assumed as a parabolic function by the square root approximation [31]-[32]. Fermi-Dirac integral is sufficient to obtain the carrier statistic in the parabolic state, while in the non-parabolic function; a disparate type of Fermi integral is needed [31].

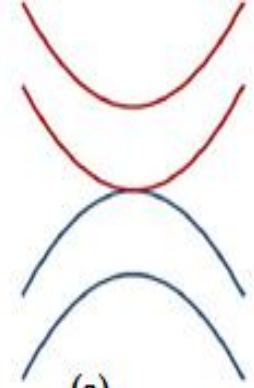

(a)

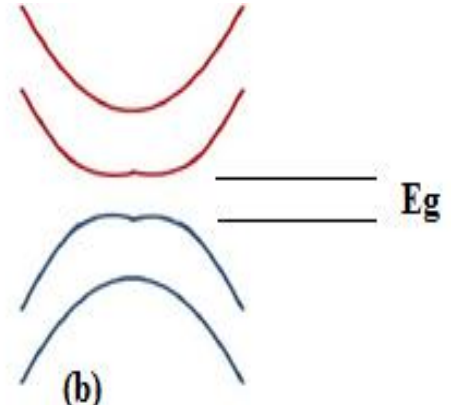

(b)

Figure 5. Schematic view of BLG with AB stacking near the Dirac points for: a) unbiased BLG $(V=0)$ and b) biased $B L G(V \neq 0)$

AA stacking of BLG always has a metallic properties, while AB stacking of BLG under special position (perpendicular voltage) has semiconductor properties. So, in proposed model we focused on the electronic properties of $\mathrm{AB}$ stacking of BLG. For the biased BLG, the energy dispersion relation is given by [33]-[34].

$$
E(k)=\frac{V_{1}+V_{2}}{2} \pm \sqrt{k^{2}+\frac{V^{2}}{4}+\frac{t_{\perp}^{2}}{2} \pm \frac{1}{2} \sqrt{4\left(V^{2}+t_{\perp}^{2}\right) k^{2}+t_{\perp}{ }^{4}}}
$$

Where $k=\left(\frac{2 m^{*}\left(E-E_{c}\right)}{\hbar^{2}}\right)^{\frac{1}{2}}+k_{g}, \mathrm{~V}_{1}$ and $\mathrm{V}_{2}$ are the applied voltages to the first and second layers respectively and $\mathrm{V}$ is the potential difference between the two layers of BLG $\left(\mathrm{V}=\mathrm{V}_{1}-\mathrm{V}_{2}\right)$. The form of Fermi surface for $(\mathrm{V} \neq 0)$ is dissimilar from $(\mathrm{V}=0)$. For the smallest band gap, wave vector of BLG as the focus of this study is adopted as [35]:

$$
k_{g}=\frac{V}{2 v_{f} \hbar} \sqrt{\frac{V^{2}+2 t_{\perp}^{2}}{V^{2}+t_{\perp}^{2}}}
$$

Where $v_{F}=(\sqrt{2} / 3) a t / \hbar \approx 1 \times 10^{6} \mathrm{~m} \cdot \mathrm{s}^{-1}$ is the Fermi velocity and $\hbar$ is the reduced Planck's constant. So, the energy dispersion of BLG near $\mathrm{k}_{\mathrm{g}}$ becomes [35]:

$$
E(k)=\frac{E_{g}}{2}+\frac{\hbar^{2}}{2 m^{*}}\left(|k|-k_{g}\right)^{2}+\frac{V_{1}+V_{2}}{2}
$$

Where $m^{*}=\frac{t_{\perp}\left(V^{2}+t_{\perp}{ }^{2}\right)^{\frac{3}{2}}}{2 V\left(V^{2}+2 t_{\perp}{ }^{2}\right)} \frac{1}{v_{F}^{2}}$ is the effective mass which is a function of applied voltage between the two layers. The effective mass of the free electron in the crystal lattice is different from $\mathrm{m}_{0}$. Based on the perpendicular electric voltage; the effective mass of the free electron in the crystal lattice is either higher or lower than $\mathrm{m}_{0}$ for the carriers in BLG lattice. It is notable that in a solid structure, electrons move around randomly without any applied electric field. On the other hand, each electron can be controlled and accelerated using an applied electric field. Due to the high electron transport, the BLG FET channel is assumed to be completely ballistic. Thus, the electrons would be accelerated to achieve faster velocity.

The Density of States (DOS) in each state of energy defines the number of states with the probability that the states are available to be occupied by electrons in any energy interval. Investigating the 
electronic properties of biased and unbiased BLG can be achieved by studying the DOS, so it can be written as:

$$
\operatorname{Dos}=\frac{\Delta n}{A \Delta E}=\left(\frac{\hbar^{2}}{m^{*}} \frac{\left(k-k_{g}\right)}{k} 2 \pi\right)^{-1}
$$

Where $k=k_{x} \hat{i}+k_{x} \hat{j}$ and A parameter shows that the DOS is dependent on the size of the BLG. According to Equation 4, the DOS increases as $\mathrm{V}$ rises.

In the conductance calculation, we need to find the number of sub-bands instead of DOS. The number of sub-bands, M (E), at applied energy can be considered near the wave vector, which is dependent on the sub band's position. By the derivation of the wave vector $k$ over the energy $E(\mathrm{dk} / \mathrm{dE})$, the number of sub bands as a summation over $\mathrm{k}$ space is written as [36]:

$$
M(E)=\frac{\Delta E}{l \Delta k}
$$

Where $\mathrm{L}$ is the length of the BLG channel, $\Delta k=\frac{\Delta k_{x} \Delta k_{y}}{2 \pi k}$ is the wave vector variation, $\Delta k_{x}=\frac{2 \pi}{l_{x}}$ and $\Delta k_{y}=\frac{2 \pi}{l_{y}}$ are the directions of wave vectors in $\mathrm{x}$ and $\mathrm{y}$, respectively. Because of the spin degeneracy in BLG, the number of BLG conducting channels is given as [36]:

$$
M(E)=\frac{2 \Delta E}{l \Delta k}
$$

It is known that the channel length of BLG has a strong effect on its conductivity. Based on the energy dispersion of Equation 3 and the effective-mass equation, we can write:

$$
\Delta E=\frac{\hbar}{m^{*}}\left(k-k_{g}\right) \Delta k
$$

Considering the wave vector, we have:

$$
M(E)=\frac{2 \hbar}{l \times m^{*}}\left(\frac{2 m^{*}\left(E-E_{c}\right)}{\hbar^{2}}\right)^{\frac{1}{2}}
$$

Where $E_{c}=\frac{E_{g}}{2}+\frac{V_{1}+V_{2}}{2}$. The ratio of the conductance to gate voltage is a basic constant, which is dependent on the Planck's constant and electron charge in bulk graphene. Thus, it is defined and calculated for the minimum conductivity by $G_{0}=\frac{2 q^{2}}{h}$.

Although the minimum conductance of BLG is two times as much as this quantity, the levels of up spin and down spin, which are located in the small channels naturally, have the energy similar to a degenerate level. The conductance of large channel in graphene materials will be obtained based on the ohmic scaling law by the Landauer formula; however, the conductance in nanoscale devices can be written by two parameters; firstly, conductance related to the width nonlinearly which depends on the number of sub-bands called quantizing parameter and secondly, interface resistance which is independent of the length.

$$
G=\frac{2 q^{2}}{h} \int_{-\infty}^{+\infty} d E M(E) T(E)\left(-\frac{d f}{d E}\right)
$$


Without scattering, electrons in ballistic transport behave according to the second law of Newton for motion of a particle at non-relativistic speeds. Thus, the electrical resistivity can be neglected in a ballistic channel of BLG due to the lack of scattering for electron transport [37]-[38]. In Equation 9, T (E) is the average probability of transmission electron in channel from one electrode to the other electrode because the assumed ballistic channel of this parameter is equal to one [39]. According to the number of sub-bands in Equation 8 and Fermi-Dirac distribution function of conductance in Equation 9, the conductance of 2D BLG with $\mathrm{AB}$ stacking can be obtained by:

$$
G=\frac{2 q^{2}}{h} \int_{-\infty}^{+\infty} d E \frac{2 \hbar}{l \times m^{*}}\left(\frac{2 m^{*}\left(E-E_{c}\right)}{\hbar^{2}}\right)^{\frac{1}{2}}\left(-\frac{d\left(\frac{1}{1+e^{\frac{E-E_{F}}{k_{B} T}}}\right)}{d E}\right)
$$

In order to simplify the conductance of equation 10, it was assumed that $x=\frac{E-E_{g}}{k_{B} T}$ and Fermi-Dirac distribution function is inserted instead of the number of sub-bands in BLG. So it is modified by:

$$
G_{B G}=\sqrt{\frac{32}{m^{*}}} \frac{q^{2}}{h l}\left(\frac{\sqrt{k_{B} T \pi}}{2} \mathfrak{J}_{-\frac{1}{2}}(\eta)-\frac{\sqrt{\eta \times k_{B} T}}{2}\right)
$$

Where $\mathfrak{I}_{-\frac{1}{2}}(\eta)$ is the Fermi-Dirac integral of orders -0.5 and normalized Fermi energy is $\eta=\frac{E_{F}-E_{g}}{k_{B} T}$.

\section{RESULTS AND ANALYSIS}

We have established a method of evaluating the perpendicular electric field effect on the resistance of BLG using potential difference values in the proposed model shown in Figure 6. Furthermore, the resistance-changing as a result of external electric field altering is different for various gate voltages. In other words, the resistance increases as the gate voltage rises. Increasing the electric field makes the larger bangap, so by focusing on the proposed model, it is evident that the resistance of BLG tends to increase as the band gap increases. The changes of BLG resistance with regard to gate voltage is shown for different values of bandgap in Figure 7 as well.

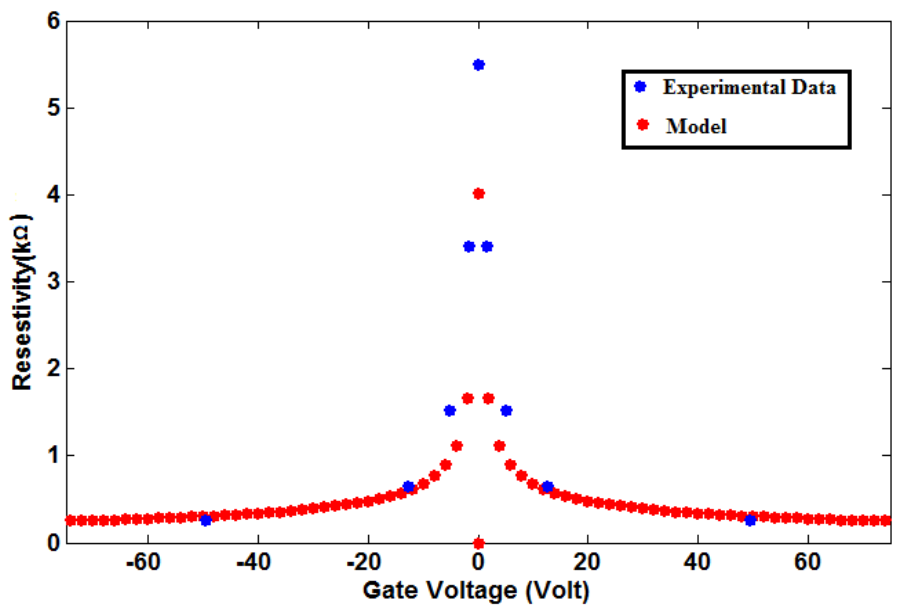

Figure 6. The comparison of resistance of BLG respect to gate voltage with modelling and experimental data [40] 


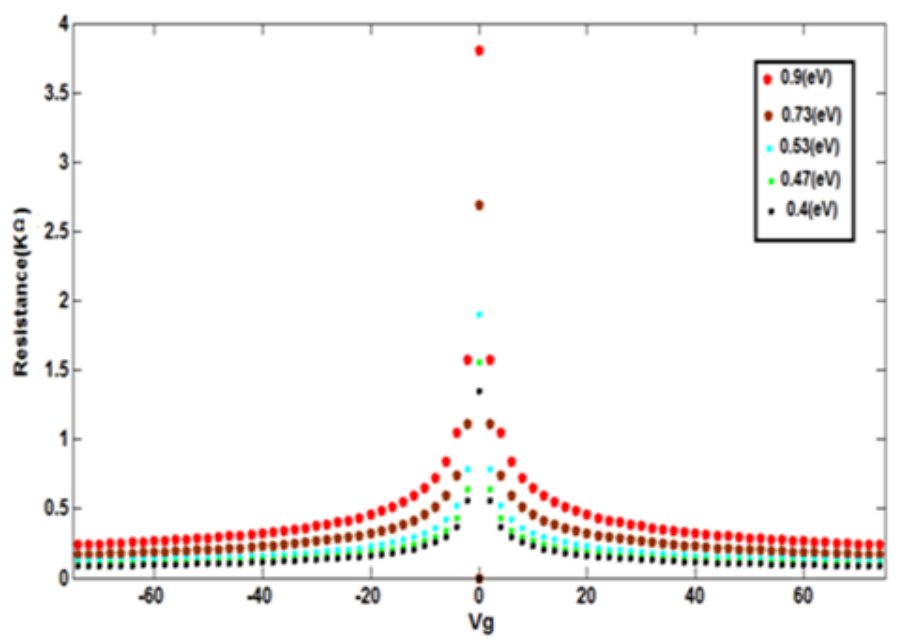

Figure 7. Effect of external voltage on resistance of BLG

It is also evident that the resistance of BLG for higher values of gate voltage $\left(\mathrm{V}_{\mathrm{g}}\right)$ increases. In addition, resistance shows a similar action regarding the $\mathrm{V}_{\mathrm{g}}$ for various values of perpendicular applied voltage. Based on the simulation results, it is concluded that the BLG band gap and resistance can be controlled by electric field. Moreover, the conductance decreases when the band gap is induced by applying an external perpendicular electric field.

The conductance of FET channel is dependent on the graphene structure and the environment conditions specially temperature. As shown in Figure 8 the conductance-changing ratio as a result of temperature altering is different for various gate voltages as well. By focusing on proposed model, it is evident that temperature can affect on the conductance of BLG. In the conductance model, the declining of BLG conductance, when temperature increases. In addition, the conductance curve is almost symmetric near $\mathrm{V}_{\mathrm{CNP}}\left(\mathrm{V}_{\mathrm{CNP}}\right.$ is a point of $\mathrm{V}_{\mathrm{g}}$ which conductance in that point is minimum value or is turning point), while at higher temperatures the conductance of BLG does not change sensible.

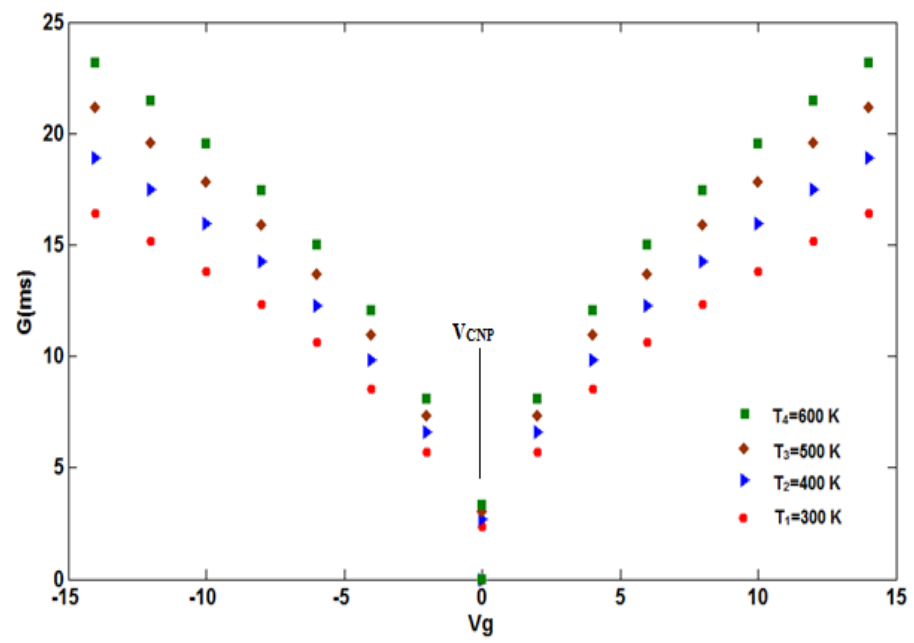

Figure 8. BLG conductance for different temperature

\section{CONCLUSION}

Different layers of graphene have various electronic properties. A perpendicular external voltage has been applied between $1^{\text {st }}$ and $2^{\text {nd }}$ layers of bilayer graphene to provide a band gap and modify its electronic properties from metallic to semiconducting. In the current study, an analytical model has been proposed for the $\mathrm{R}-\mathrm{V}_{\mathrm{g}}$ characteristic and conductance of bilayer graphene-based FET. In this research, the BLG 
conductance respect to $\mathrm{V}_{\mathrm{g}}$ is investigated and it is notable that $\mathrm{BG}$ band gap and resistance can be controlled by applying an external perpendicular electric field. Also, increasing temperature can effect in the resistance of BLG unless for charge natural point. Ultimately, it is remarkable that BLG conductance for high values of temperature is increased.

\section{REFERENCES}

[1] Morsin, M. and Y. Yusof, The Ab-initio Study of Bulk Single Layer Defected Graphene Towards Graphene Device. International Journal of Electrical and Computer Engineering (IJECE), 2017. 7(3).

[2] Morsin, M., et al., Characterization of Defect Induced Multilayer Graphene. International Journal of Electrical and Computer Engineering (IJECE), 2017. 7(3).

[3] Mehrdel, B., A.A. Aziz, and M.H. Ghadiri, Effect of Device Variables on Surface Potential and Threshold Voltage in DG-GNRFET. International Journal of Electrical and Computer Engineering, 2015. 5(5).

[4] Yao, Y.G., et al., Controlled Growth of Multilayer, Few-Layer, and Single-Layer Graphene on Metal Substrates. Journal of Physical Chemistry C, 2011. 115(13): p. 5232-5238.

[5] Barbier, M., et al., Bilayer graphene with single and multiple electrostatic barriers: Band structure and transmission. Physical Review B, 2009. 79(15).

[6] Das, A., B. Chakraborty, and A.K. Sood, PROBING SINGLE AND BILAYER GRAPHENE FIELD EFFECT TRANSISTORS BY RAMAN SPECTROSCOPY. Modern Physics Letters B, 2011. 25(8): p. 511-535.

[7] Mousavi, S., Ahmadi, MT,Webb, JF,Sadeghi, H.,Nilghaz, A. Amin, A. Johari, Z.Ismail, R., Bilayer Graphene Nanoribbon Carrier Statistics in the Degenerate Regime. AIP Conference Proceedings, 2011. 1337: p. 180.

[8] Mousavi, S.M., et al. Bilayer Graphene nanoribbon conductance model in parabolic band structure. 2010: IEEE.

[9] Mousavi, S.M.A., M.T.Sadeghi, H.Nilghaz, A.Amin, A.Johari, Z.Ismail, R., Bilayer Graphene Nanoribbon Carrier Statistic in Degenerate and Non Degenerate Limit. Journal of Computational and Theoretical Nanoscience, 2011. 8(10): p. 2029-2032.

[10] Rahmani, M., et al. Current-voltage modeling of Bilayer Graphene Nanoribbon Schottky Diode. 2011: IEEE.

[11] Sadeghi, H.A., MT Ishak, BI Mousavi, SM Ismail, R., Ballistic Conductance Model of Bilayer Graphene Nanoribbon (BGN). Journal of Computational and Theoretical Nanoscience, 2011. 8(10): p. 1993-1998.

[12] Sadeghi, H., et al., Carrier Statistics Model for a Bilayer Graphene Nanoribbon in the Nondegenerate Regime. AIP Conference Proceedings, 2011. 1337: p. 184.

[13] Sadeghi, H., et al., Biased Voltage Boundary Condition to Operate Bilayer Graphene in the Insulating Region.

[14] V. Schmidt, J.V. Wittemann, S. Senz, U. Goesele, Silicon nanowires: a review on aspects of their growth and their electrical properties. Adv. Mater. 21, 2681-2702 (2009).

[15] Chen, Y.-C. et al. Tuning the band gap of graphene nanoribbons synthesized from molecular precursors. ACS Nano 7, 6123-6128 (2013).

[16] Huang, B., et al., Adsorption of gas molecules on graphene nanoribbons and its implication for nanoscale molecule sensor. Journal of Physical Chemistry C, 2008. 112(35): p. 13442-13446.

[17] Kimouche, A. et al. Ultra-narrow metallic armchair graphene nanoribbons. Nat. Commun. 6, 10177 (2015).

[18] Lu, Y.H., et al., Effects of edge passivation by hydrogen on electronic structure of armchair graphene nanoribbon and band gap engineering. Applied Physics Letters, 2009. 94(12).

[19] Moradian, R., Y. Mohammadi, and N. Ghobadi, Investigation of gas sensing properties of armchair graphene nanoribbons. Journal of Physics-Condensed Matter, 2008. 20(42).

[20] Cai, J. et al. Graphene nanoribbon heterojunctions. Nat. Nanotechnol. 9, 896-900 (2014).

[21] Jung, J., Nonlocal exchange effects in zigzag-edge magnetism of neutral graphene nanoribbons. Physical Review B, 2011. 83(16).

[22] K. Natori, "Scaling Limit of the MOS Transistor-A Ballistic MOSFET", IEICE Trans. Electron. E84-C, no. 8, 1029 (2001).

[23] Arghavan, S. and A.V. Singh, Effects of van der Waals interactions on the nonlinear vibration of multi-layered graphene sheets. Journal of Physics D-Applied Physics, 2012. 45(45).

[24] Shioya, H., et al., Gate tunable non-linear currents in bilayer graphene diodes. Applied Physics Letters, 2012. 100(3): p. 033113-4.

[25] Falkovsky, L.A., Gate-tunable bandgap in bilayer graphene. Journal of Experimental and Theoretical Physics, 2010. 110(2): p. 319-324

[26] Son, Y.-W., M.L. Cohen, and S.G. Louie, Energy Gaps in Graphene Nanoribbons. Physical Review Letters, 2006. 97(21): p. 216803

[27] Zhang, W., et al., Opening an Electrical Band Gap of Bilayer Graphene with Molecular Doping. ACS Nano, 2011. 5(9): p. 7517-7524.

[28] Zhang, Y., et al., Direct observation of a widely tunable bandgap in bilayer graphene. Nature, 2009. 459(7248): p. 820-823.

[29] Ando, T. and M. Koshino, Field effects on optical phonons in bilayer graphene. J. Phys. Soc. Jpn. 78, 034709/1-8 (2009).

[30] Eduardo, V.C., et al., Electronic properties of a biased graphene bilayer. Journal of Physics: Condensed Matter, 2010. 22(17): p. 175503.

[31] Zaharah Johari, M.T.A., Desmond Chek Chang Yih, N. Aziziah Amin, and Razali Ismail, Modelling of Graphene Nanoribbon Fermi Energy. Journal of Nanomaterials. 2011, Article ID 909347, 6 pages. 
[32] Narendar, S., Buckling analysis of micro-/nano-scale plates based on two-variable refined plate theory incorporating nonlocal scale effects. Composite Structures, 2011. 93(12): p. 3093-3103.

[33] J. Nilsson, A.H.C.N., F. Guinea, N. M. R. Peres, Electronic properties of bilayer and multilayer graphene. Phys. Rev. B. 78 (2008) 045405. doi:10.1103/PhysRevB.78.045405.

[34] J. Nilsson, A.H.C.N., F. Guinea, N. M. R. Peres, Phys. Rev. B, Transmission through a biased graphene bilayer barrier. 76 (2007) 165416. doi:10.1103/PhysRevB.76.165416.

[35] J. Nilsson, A.H.C.N., F. Guinea, N. M. R. Peres Electronic properties of graphene multilayers. Phys. Rev. Lett.. 97 (2006) 266801 doi:10.1103/PhysRevLett.97.266801.

[36] J. Nilsson, A.H.C.N., F. Guinea, N. M. R. Peres, Electronic properties of graphene multilayers. Phys. Rev. Lett. 97 (2006) 266801 doi:10.1103/PhysRevLett.97.266801.

[37] Levinshtein, Michael E., Rumyantsev, Sergey L., and Shur, Michael S., Properties of Advanced Semiconductor Materials. New York, John Wiley \& Sons, 2001.

[38] Abergel, D.S.L., et al., Properties of graphene: a theoretical perspective. Advances in Physics, 2010. 59(4): p. 261482.

[39] Avila, A.F., A.S. Neto, and H. Nascimento Junior, Hybrid nanocomposites for mid-range ballistic protection. International Journal of Impact Engineering, 2011. 38(8-9): p. 669-676.

[40] Datta, S., Electronic Transport in Mesoscopic Systems. Cambridge University Press, Cambridge, UK (2002).

[41] A. V.-G. Ferreira, J. Nilsson, J. Mucciolo, E. R. Peres, N. M. R. C. Neto, A. H. U., Unified description of the dc conductivity of monolayer and bilayer graphene at finite densities based on resonant scatterers. Phys. Rev. B 83, 16 (2011). 